\title{
Optical Vortices Sharp Focusing by Silicon Ring Gratings Using High-Performance Computer Systems
}

\author{
Dmitry SAVELYEV ${ }^{\mathrm{a}, \mathrm{b}, 1}$

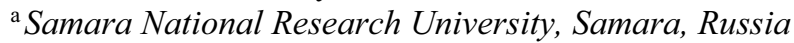 \\ b IPSI RAS - Branch of the FSRC "Crystallography and Photonics” RAS, Samara, \\ Russia
}

\begin{abstract}
The diffraction of vortex laser beams with circular polarization (with different direction of polarization rotation) by silicon ring gratings was investigated in this paper. The silicon diffractive axicons with different numerical apertures (NA) were considered as such ring gratings. The considered diffractive axicons are compared with single silicon circular protrusion (cylinder). The finite difference time domain method was used for Light propagation (3D) through the proposed silicon ring gratings and silicon cylinder. The possibility of subwavelength focusing by varying the height of the elements is demonstrated. In particular, it is numerically shown that a silicon cylinder forms a light spot with the minimum size (intensity) of the longitudinal component of the electric field FWHM is $0.32 \lambda$.
\end{abstract}

Keywords. Silicon photonics, subwavelength ring gratings, optical vortices, FDTD

\section{Introduction}

The silicon photonics has attracted more attention from researchers in the past few decades [1-5]. This is due to a number of reasons, including the cheaper implementation of optical devices using standard semiconductor manufacturing technologies [2] and miniaturization of solutions for light transmission through optical fibers [3]. It is also possible to obtain a high integration density due to the large refractive index of silicon [1]. Photonics in general and silicon photonics in particular are becoming a promising alternative for the implementation of new accelerators for deep learning that can use light, both for communication and for computing, thanks to such advantages as high speed, wide bandwidth and parallelism [6,7].

A number of classical structures in the construction of crossing silicon waveguides are well known, such as subwavelength grating, shaped taper, holey subwavelength grating [3]. In particular, the methodology for designing silicon based waveguide Bragg gratings (WBGs) in detail discusses the paper [8]. And one of the ways to increase the number of channels for data transmission is the introduction of higher order modes in mode-division-multiplexed (MDM) systems, which also allows for

${ }^{1}$ Corresponding Author: Dmitry Savelyev, Department of Technical Cybernetics, Samara National Research University, 443086 Samara, Russia; E-mail: dmitrey.savelyev@yandex.ru. 
increased device design flexibility [9]. One of the promising methods is channel thickening at modes with different orders of the vortex phase singularity $[10,11]$.

It should be noted that for efficient light beams insertion into a small diameter optical fiber can be used sharp focusing [12]. The vortex modes are scale invariant at the exit from the fiber (using diffraction microstructures) and a decrease in the radiation divergence at the exit of an optical fiber can be obtained by structuring the exit end of a fiber with a ring structure [13]. Such a ring grating (for example, a diffractive axicon) with a shorter period (larger numerical aperture of the axicon) makes it possible to achieve sharper focusing than an aplanatic lens with the same numerical aperture of the system $[14,15]$. The diameter of the central spot at full width at half maximum intensity (FWHM) of such an element is 0.36 wavelength divided by the numerical aperture [16]. The possibility of manufacturing microaxicons expands the area of their application $[15,17]$ and allows them to be used to form structured light fields.

The introduction of vortex phase singularity into the incident beam makes it possible to enhance the longitudinal component of homogeneous-polarized laser beams on the optical axis in the focal region [18]. So, it which makes it possible to change the diffraction pattern due to the redistribution of energy between the components of the electromagnetic field $[19,20]$. This was shown earlier for focusing elements with a refractive index $n=1.46[19,20]$, but an increase in the contribution of the longitudinal component to the overall intensity pattern on the optical axis can be achieved [18] with an increasing of the refractive index.

The diffraction of vortex Gaussian beams by silicon ring gratings, representing diffraction axicons with different numerical apertures study in this paper. A separate protrusion in the form of a circular cylinder, close in size to the size of the central part of the diffractive axicons, was also considered. The effect of changing the height of the considered optical elements on subwavelength focusing is investigated. Numerical calculations of laser propagation (3D) were performed using high performance computer systems (computing cluster with a capacity of 800 Gflops) with the finite difference time domain (FDTD) method [21,22].

\section{Investigation of the Laguerre-Gauss Modes $(1,0)$ Diffraction by Silicon Cylinder}

Modeling parameters: the size of the computational domain $\mathrm{x}, \mathrm{y}, \mathrm{z}$ in the range $[-5.8 \lambda$; $5.8 \lambda]$, radiation wavelength $\lambda=1.55 \mu \mathrm{m}$, the spatial sampling step is $\lambda / 30$, the time sampling step is $\lambda /(60 \mathrm{c})$, where $\mathrm{c}$ is the speed of light, and the thickness of the absorbing layer PML is $1.16 \lambda$ (on all sides surrounding the computational domain). The source was located inside the substrate, which occupies the entire space below the relief and is partially embedded in the PML layer. The refractive index of the element $n$ is 3.47. The Laguerre-Gauss mode $(1,0)$ (optical vortex) was considered as the input laser radiation:

$$
G L_{01}(r, \varphi, z)=\left(\frac{\sqrt{2} r}{\sigma(z)}\right) \exp [i k z-i 2 \eta(z)] \exp \left[\frac{i \pi r^{2}}{\lambda R(z)}\right] \exp \left[-\frac{r^{2}}{\sigma^{2}(z)}\right] \exp (i \varphi)
$$


where $\varphi=\operatorname{arctg}(y / x), \eta(z)=\operatorname{arctg}\left(z / z_{0}\right), r^{2}=x^{2}+y^{2}, R(z)=\mathrm{z}\left(1+z_{0}^{2} / z^{2}\right)$ is the radius of curvature of the light field parabolic front, $z_{0}=\pi \sigma_{0}^{2} / \lambda$ is the confocal parameter, $\sigma(z)$ is the effective beam radius, and $\lambda$ is the wavelength.

The height $h$ of the binary element relief (even if it is a separate protrusion), corresponding to the phase jump $\pi$ radians, for the selected refractive index is as follows:

$$
h=\frac{\pi}{k(n-1)} \approx 0.2 \lambda,
$$

where $\mathrm{k}=2 \pi / \lambda$ - wave number, $\lambda$ - wavelength of laser radiation, $\mathrm{n}$ - refractive index.

The FDTD method is currently one of the most common methods for solving Maxwell's equations written in differential form. The Maxwell's equations using the FDTD method are solved by discretizing using central differences in time and space, and then solving them numerically [23]. The Meep package (was developed by a research group of scientists from the Massachusetts University of Technology) was used for numerical simulation with FDTD method [24]. The Meep package uses standard Yee grid sampling.

The results of a study on the passage of the Laguerre-Gauss mode $(1,0)$ through the silicon cylinder with a radius of $r=2 \lambda$ are shown in the Figure 1 .
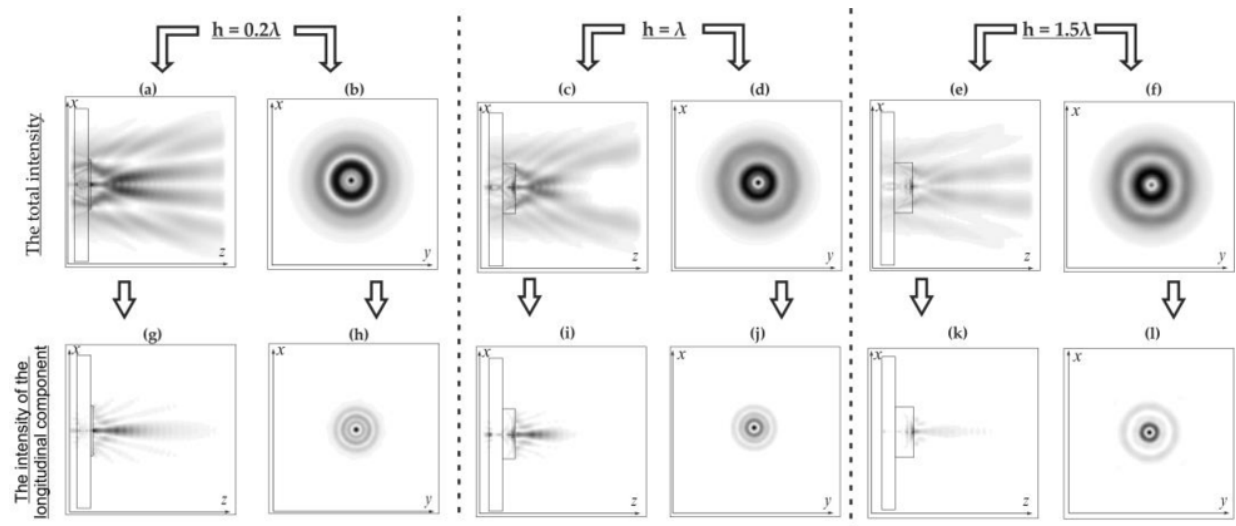

Figure 1. The optical vortex diffraction by circular protrusion ("-" - circular polarization): for total intensity of the electric field at $\mathrm{h}=0.2 \lambda-(\mathrm{a}),(\mathrm{b})$, at $\mathrm{h}=\lambda-(\mathrm{c}),(\mathrm{d})$, at $\mathrm{h}=1.5 \lambda-(\mathrm{e}),(\mathrm{f})$; and the intensity of the electric field longitudinal component at $\mathrm{h}=0.2 \lambda-(\mathrm{g}),(\mathrm{h})$, at $\mathrm{h}=\lambda-(\mathrm{i}),(\mathrm{j})$, at $\mathrm{h}=1.5 \lambda-(\mathrm{k}),(\mathrm{l})$.

The addition of an optical vortex significantly changes the focal pattern and the direction of rotation of circular polarization becomes important $[18,19]$. At the second optical vortex order and higher for "-" - circular polarization (the sign of circular polarization is opposite to the sign of the introduced vortex phase singularity), a shadow round light spot was formed. So, the first order of an optical vortex in an incident beam considered in this paper. The polarization, the direction of which coincides with the direction of the vortex phase singularity, will be called "+" - circular polarization. As expected, a zero value (Figure 2) for a central focal spot indicates "+" circular polarization, and "-" - circular polarization indicates by non-zero value [15]. 

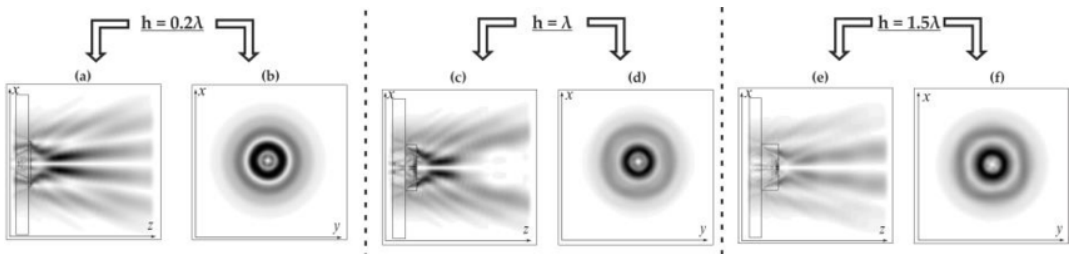

Figure 2. The optical vortex diffraction by circular protrusion ("+" - circular polarization), total intensity of the electric field at $h=0.2 \lambda-(a),(b)$, at $h=\lambda-(c),(d)$, at $h=1.5 \lambda-(e),(f)$.

The focal spot size on the optical axis was estimated at FWHM of the all intensity and FWHM of the intensity of the electric field longitudinal component (FWHMz). The distance from the edge of the microrelief to the section plane is denoted as $d$. The transverse diffraction patterns (xy plane) considered at a distance of $d=0.5 \lambda$. The cylinder height increasing resulted in better focusing: $\mathrm{FWHM}=0.45 \lambda$ at $\mathrm{h}=0.2 \lambda$ $(\mathrm{FWHMz}=0.37 \lambda), \mathrm{FWHM}=0.37 \lambda$ at $\mathrm{h}=\lambda(\mathrm{FWHMz}=0.35 \lambda)$ and $\mathrm{FWHM}=0.36 \lambda$ at $\mathrm{h}=1.5 \lambda(\mathrm{FWHMz}=0.32 \lambda)$.

\section{Investigation of the Laguerre-Gauss Modes (1,0) Diffraction by Axicons}

In this section, studies of the influence of a change in the relief height on the diffraction pattern in the near zone are continued. The simulation parameters are the same as in the previous section. The results of a study on the passage input beams through the diffractive axicons with NA $=0.25$ (grating period $4 \lambda$ ) and NA $=0.95$ (grating period $1.05 \lambda$ ) are shown in the Figure 3 and Figure 4.
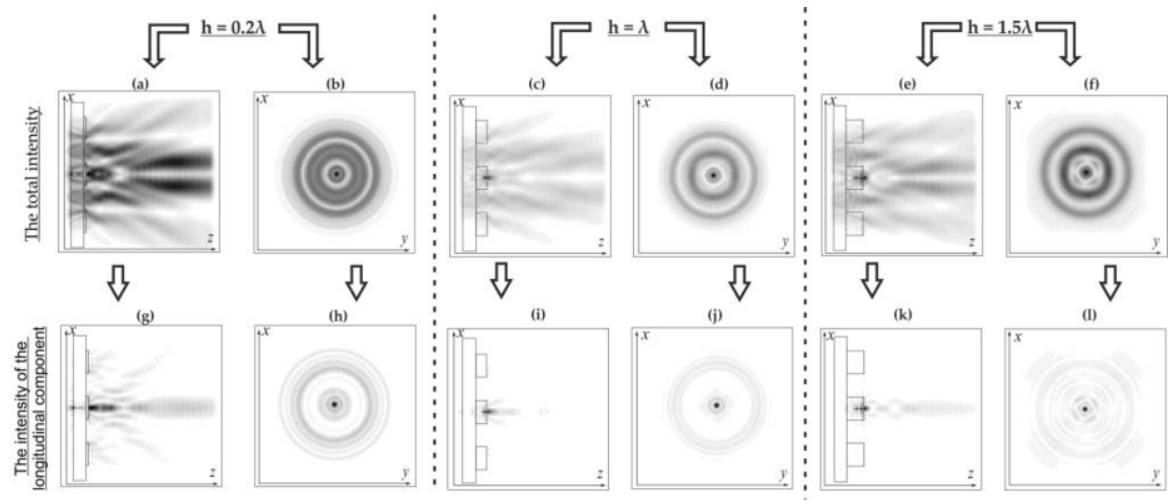

Figure 3. The optical vortex diffraction by an axicon with NA $=0.25$ ("-" - circular polarization): for total intensity of the electric field at $h=0.2 \lambda-(a),(b)$, at $h=\lambda-(c),(d)$, at $h=1.5 \lambda-(e)$, (f); and the intensity of the electric field longitudinal component at $\mathrm{h}=0.2 \lambda-(\mathrm{g})$, (h), at $\mathrm{h}=\lambda-(\mathrm{i}),(\mathrm{j})$, at $\mathrm{h}=1.5 \lambda-(\mathrm{k}),(\mathrm{l})$.

The transverse diffraction patterns (xy plane) considered at a distance of $d=0.2 \lambda$ for diffractive axicon with NA $=0.25$ (and for case $\mathrm{h}=1.5 \lambda$ for $\mathrm{NA}=0.95$ ) and $\mathrm{d}=$ $0.5 \lambda$ for diffractive axicon with NA $=0.95(\mathrm{~h}=0.2 \lambda$ and $\mathrm{h}=\lambda)$. The following results were obtained: for diffractive axicon with NA $=0.25, \mathrm{FWHM}=0.61 \lambda$ at $\mathrm{h}=0.2 \lambda$ $(\mathrm{FWHMz}=0.46 \lambda), \mathrm{FWHM}=0.48 \lambda$ at $\mathrm{h}=\lambda(\mathrm{FWHMz}=0.43 \lambda)$ and $\mathrm{FWHM}=0.86 \lambda$ at $\mathrm{h}=1.5 \lambda(\mathrm{FWHMz}=0.34 \lambda)$; for diffractive axicon with $\mathrm{NA}=0.95, \mathrm{FWHM}=0.48 \lambda$ at $\mathrm{h}=0.2 \lambda(\mathrm{FWHMz}=0.44 \lambda), \mathrm{FWHM}=0.47 \lambda$ at $\mathrm{h}=\lambda(\mathrm{FWHMz}=0.43 \lambda)$ and $\mathrm{FWHM}=$ $0.67 \lambda$ at $\mathrm{h}=1.5 \lambda(\mathrm{FWHMz}=0.36 \lambda)$. The contribution of the longitudinal component of 
the electric field to the total intensity decreases with an increase in the height of the aaxicon relief, despite a decrease in the size of the focal spot (only the longitudinal component). In addition, the size of the central focal spot begins to be influenced by the transverse components of the electric field, leading to its broadening.

It should also be noted that for the axicon with $\mathrm{NA}=0.95$, strong side lobes are formed for the case $h=1.5 \lambda$; however, at a lower height, it gives better focusing compared to a smaller numerical aperture. Also note that for a silicon cylinder with $\mathrm{r}=$ $2 \lambda$, a narrower focal spot on optical axis was obtained (FWHM $=0.37 \lambda$ at $\mathrm{h}=\lambda$ and $\mathrm{FWHM}=0.36 \lambda$ at $\mathrm{h}=1.5 \lambda)$, and at $\mathrm{h}=0.2 \lambda$ the size of the focal spot is comparable with axicons result.

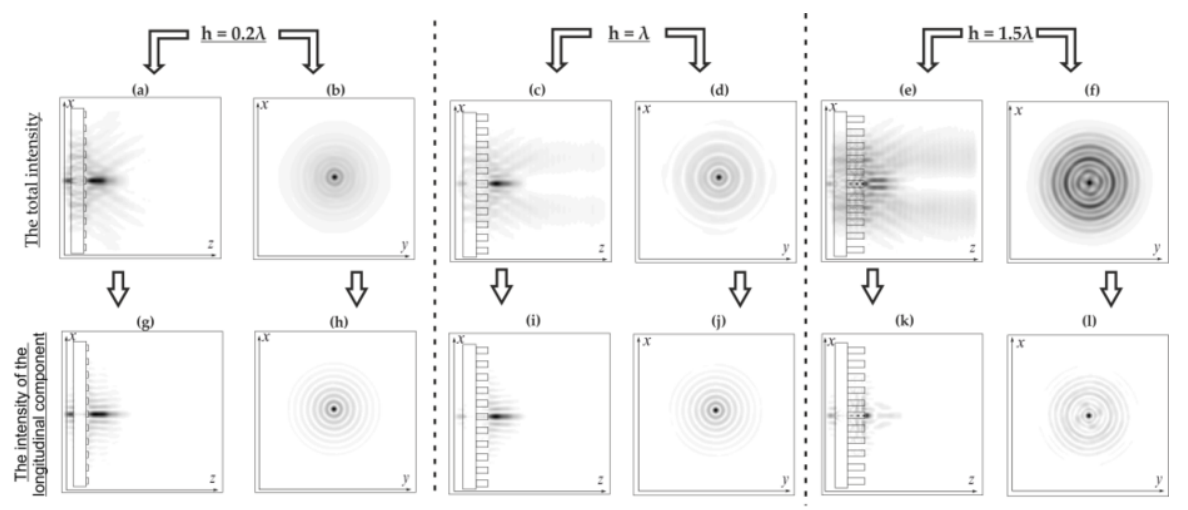

Figure 4. The optical vortex diffraction by an axicon with NA $=0.95("-"$ - circular polarization): for total intensity of the electric field at $\mathrm{h}=0.2 \lambda-(\mathrm{a}),(\mathrm{b})$, at $\mathrm{h}=\lambda-(\mathrm{c}),(\mathrm{d})$, at $\mathrm{h}=1.5 \lambda-(\mathrm{e})$, (f); and the intensity of the electric field longitudinal component at $\mathrm{h}=0.2 \lambda-(\mathrm{g}),(\mathrm{h})$, at $\mathrm{h}=\lambda-(\mathrm{i}),(\mathrm{j})$, at $\mathrm{h}=1.5 \lambda-(\mathrm{k}),(\mathrm{l})$.

\section{Conclusions}

The diffraction of vortex laser beams with circular polarization by silicon ring gratings was investigated in this paper. The silicon cylinder focusing is compared with diffractive axicons with different grating periods and shown, that the result of focusing with a circular cylinder with a radius of $r=2 \lambda$ was better than focusing by a diffractive axicon with grating period $4 \lambda(\mathrm{NA}=0.25)$ and comparable to focusing by a diffractive axicon with grating period $1.05 \lambda(\mathrm{NA}=0.95)$ at low relief height.

The enhancement possibility of sub-wavelength focusing is demonstrated when the height of the elements is increasing. It is shown that in this case, the contribution of the electric field longitudinal component to the size of the central focal spot increases up to a certain height. Also shown that a silicon cylinder forms a light spot with the minimum size (intensity) of the electric field longitudinal component FWHM is $0.32 \lambda$ with $\mathrm{h}=1.5 \lambda$.

\section{Acknowledgement}

This research was funded by the Russian Science Foundation (project No. 20-7200051) in the parts «Investigation of the Laguerre-Gauss Modes $(1,0)$ Diffraction by 
Silicon Cylinder», «Investigation of the Laguerre-Gauss Modes $(1,0)$ Diffraction by Axicons»; the Ministry of Science and Higher Education and was performed under the «ERA.Net RUS plus» program and funded by RFBR, project number 20-52-76021» in the parts «Introduction», «Conclusions».

\section{References}

[1] Mu X, Wu S, Cheng L, Fu HY. Edge couplers in silicon photonic integrated circuits: A review. Applied Sciences 2020; 10(4): 1538 (29 pp.).

[2] Cheng L, Mao S, Li Z, Han Y, Fu HY. Grating couplers on silicon photonics: design principles, emerging trends and practical issues. Micromachines 2020; 11(7): 666 (25 pp.).

[3] Wu S, Mu X, Cheng L, Mao S, Fu HY. State-of-the-art and perspectives on silicon waveguide crossings: A review. Micromachines 2020; 11(3): 326 (16 pp.).

[4] Butt M, Khonina SN, Kazanskiy NL. Optical elements based on silicon photonics. Computer Optics 2019; 43(6): 1079-1083.

[5] Leonardi AA, Faro MJL, Irrera A. Silicon Nanowires Synthesis by Metal-Assisted Chemical Etching: A Review. Nanomaterials 2021; 11(2): 383 (24 pp.).

[6] Shastri BJ, et al. Photonics for artificial intelligence and neuromorphic computing. Nature Photonics 2021; 15(2): 102-114.

[7] Xiang S, et al. A review: Photonics devices, architectures, and algorithms for optical neural computing. Journal of Semiconductors 2021; 42(2): 023105.

[8] Kaushal S, et al. Optical signal processing based on silicon photonics waveguide Bragg gratings. Frontiers of Optoelectronics 2018; 11(2): 163-188.

[9] Li C, Liu D, Dai D. Multimode silicon photonics. Nanophotonics 2018; 8(2): 227-247.

[10] Bozinovic, N. et. al. Terabit-Scale Orbital Angular Momentum Mode Division Multiplexing in Fibers. Science 2013; 340(6140): 1545-1548.

[11] $\mathrm{Wu}, \mathrm{H}$, et al. All-fiber second-order optical vortex generation based on strong modulated long-period grating in a four-mode fiber. Optics Letters 2017; 42(24): 5210-5213.

[12] Savelyev DA, Khonina SN. Sharp focusing by means of binary relief at the end of the optical fiber. Optical Technologies for Telecommunications 2013; 2014; 9156: 915609 (6 pp.).

[13] Liu Y, Xu H, Stief F, Zhitenev N, Yu M. Far-field superfocusing with an optical fiber based surface plasmonic lens made of nanoscale concentric annual slits. Opt. Express 2011; 19(21): 20233-20243.

[14] Khonina SN, Savelyev DA, Kazanskiy NL. Analysis of polarisation states at sharp focusing. OptikInternational Journal for Light and Electron Optics 2016; 127(6): 3372-3378.

[15] Khonina SN, Savelyev DA, Kazanskiy NL. Vortex phase elements as detectors of polarization state. Optics Express 2015; 23(14): 17845-17859.

[16] Kalosha VP, Golub I. Toward the subdiffraction focusing limit of optical superresolution. Optics Letters 2007; 32(24): 3540-3542.

[17] Khonina S, Degtyarev S, Savelyev D, Ustinov A. Focused, evanescent, hollow, and collimated beams formed by microaxicons with different conical angles. Optics Express 2017; 25(16): 19052-19064.

[18] Khonina SN, Savelyev DA. High-aperture binary axicons for the formation of the longitudinal electric field component on the optical axis for linear and circular polarizations of the illuminating beam. Journal of Experimental and Theoretical Physics 2013; 117(4): 623-630.

[19] Savelyev DA, Khonina SN. Characteristics of sharp focusing of vortex Laguerre-Gaussian beams. Computer Optics 2015; 39(5): 654-662.

[20] Khonina SN, et al. Experimental demonstration of the generation of the longitudinal E-field component on the optical axis with high-numerical-aperture binary axicons illuminated by linearly and circularly polarized beams. Journal of Optics 2013; 15(8): 085704.

[21] Khonina S, Savelyev D. Optimization of the Optical Microelements Using High-Performance Computer Systems. Radiophysics \& Quantum Electronics 2015; 57(8-9): 650-658.

[22] Savelyev D. The investigation of the cylindrically polarized beams focusing by a diffractive axicon using high-performance computer systems. 2020 International Conference on Information Technology and Nanotechnology (ITNT), IEEE; 2020 May: 1-5.

[23] Taflove A, Hagness SC. Computational electrodynamics: the finite-difference time-domain method. Norwood, MA: Artech House; 2005. 1038 p.

[24] Oskooi AF, Roundy D, Ibanescu M, Bermel P, Joannopoulos JD, Johnson SG. MEEP: A flexible freesoftware package for electromagnetic simulations by the FDTD method. Computer Physics Communications 2010; 181(3): 687-702. 